\title{
Bimanual Interaction with Interscopic Multi-Touch Surfaces
}

\author{
Johannes Schöning $^{1}$, Frank Steinicke ${ }^{2}$, Antonio Krüger ${ }^{1}$, \\ Klaus Hinrichs ${ }^{2}$, and Dimitar Valkov ${ }^{2}$ \\ ${ }^{1}$ DFKI (German Research Center for Artifical Intelligence), Intelligent User Interfaces \\ Department, Stuhlsatzenhausweg 3, 66123 Saarbrücken, Germany \\ \{schoening, krueger\} @dfki.de \\ ${ }^{2}$ Visualization and Computer Graphics Group, Department of Computer Science University \\ of Münster, Einsteinstr. 62, 48149 Münster, Germany \\ \{frank.steinicke, khh, dimitar.valkov\}@uni-muenster.de
}

\begin{abstract}
Multi-touch interaction has received considerable attention in the last few years, in particular for natural two-dimensional (2D) interaction. However, many application areas deal with three-dimensional (3D) data and require intuitive 3D interaction techniques therefore. Indeed, virtual reality (VR) systems provide sophisticated 3D user interface, but then lack efficient 2D interaction, and are therefore rarely adopted by ordinary users or even by experts. Since multi-touch interfaces represent a good trade-off between intuitive, constrained interaction on a touch surface providing tangible feedback, and unrestricted natural interaction without any instrumentation, they have the potential to form the foundation of the next generation user interface for $2 \mathrm{D}$ as well as $3 \mathrm{D}$ interaction. In particular, stereoscopic display of 3D data provides an additional depth cue, but until now the challenges and limitations for multi-touch interaction in this context have not been considered. In this paper we present new multi-touch paradigms and interactions that combine both traditional 2D interaction and novel 3D interaction on a touch surface to form a new class of multitouch systems, which we refer to as interscopic multi-touch surfaces (iMUTS). We discuss iMUTS-based user interfaces that support interaction with 2D content displayed in monoscopic mode and 3D content usually displayed stereoscopically. In order to underline the potential of the proposed iMUTS setup, we have developed and evaluated two example interaction metaphors for different domains. First, we present intuitive navigation techniques for virtual 3D city models, and then we describe a natural metaphor for deforming volumetric datasets in a medical context.
\end{abstract}

Keywords: Multi-touch Interaction, Interscopic Interaction, 3D User Interfaces.

\section{Introduction and Related Work}

In recent years, the visualization of and the interaction with three-dimensional (3D) data has become more and more popular and widespread due to the requirements of numerous application areas. Two-dimensional (2D) desktop systems are often limited in cases in which natural and intuitive interfaces are desired. Sophisticated 3D user 


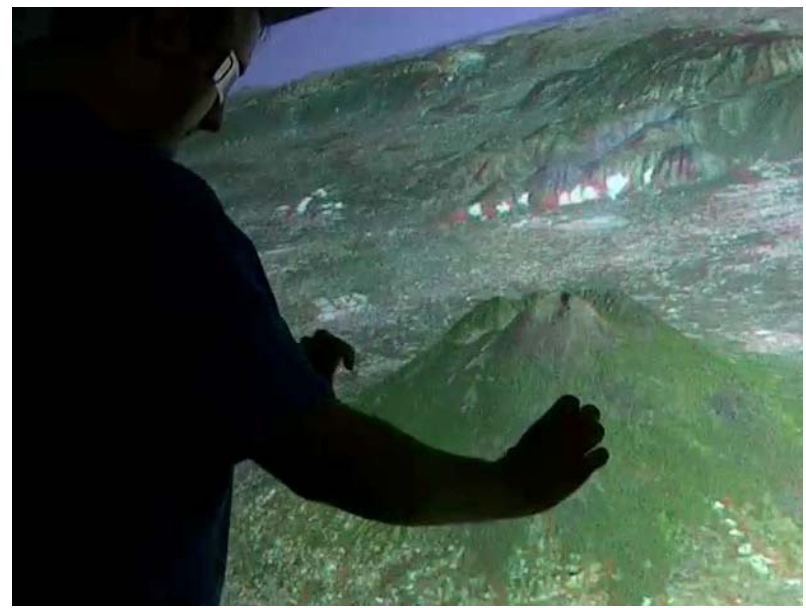

Fig. 1. A user interacting with an interscopic multi-touch surface (iMUTS) in a landscape planning scenario in which depth cues are important, e. g., in a landslide risk management scenario. The geospatial data is displayed in anaglyph stereoscopic mode.

interfaces, as they are provided by virtual reality (VR) systems consisting of stereoscopic projection and tracked input devices, are rarely adopted by ordinary users or even by experts often due to the overall complexity of the user interface. However, a major benefit of stereoscopy is binocular disparity that provides a better depth awareness. When a stereoscopic display is used, each eye of the user perceives a different perspective of the same scene. This can be achieved by either having the user wear special glasses or by using special 3D displays. For this reason, VR systems using tracking technologies and stereoscopic projections of $3 \mathrm{D}$ synthetic worlds have a great potential to support a better exploration of complex data sets. However, interaction with stereoscopic display may become difficult. For instance, in a stereoscopically displayed 3D scene, it may be hard to access distant objects [1]. This applies in particular if the interaction is restricted to a $2 \mathrm{D}$ touch surface. Objects might be displayed with different parallax paradigms, i.e., negative, zero, and positive parallax, resulting in different stereoscopic effects. Interaction with objects that are displayed with different parallaxes is still a challenging task even in VR-based environments. In addition, while the costs as well as the effort to acquire and maintain VR systems have decreased to a moderate level, these setups are only used in highly specific application scenarios within some VR laboratories. In most human-computer interaction processes, VR systems are only rarely applied by ordinary users or by experts - even when 3D tasks have to be accomplished [2]. One reason for this is the inconvenient instrumentation required to allow immersive interactions in such VR systems, i.e., the user is forced to wear stereo glasses, tracked devices, gloves etc. Furthermore, the most effective ways for humans to interact with synthetic 3D environments have not finally been determined [2, 1]. Even the WIMP (window, icon, menu, pointing device) desktop metaphor [3] has its limitations when it comes to direct manipulation of 3D data sets [4], e.g., via 3D widgets [5]. And as a matter of fact, 2D interactions are performed best with $2 \mathrm{D}$ devices usually supporting only two degrees of freedom 
(DoF) $[6,7]$. Hence, 3D user interfaces are often the wrong choice in order to accomplish tasks requiring exclusively or mainly two-dimensional control $[2,6]$. Most 3D applications also include 2D user interface elements, such as menus, texts and images, in combination with 3D content. While 3D content usually benefits from stereoscopic visualization, 2D graphical user interfaces (GUIs) items often do not have associated depth information. Therefore, interactions between monoscopic and stereoscopic elements, so-called interscopic interactions [8], have not been fully examined with special consideration of the interrelations between the elements. Due to recent developments in the entertainment market, multi-touch has received considerable attention for 2D user interfaces. Multi-touch refers to a set of interaction paradigms, which allow users to control applications with several simultaneously performed touches. While multi-touch has shown its usefulness for 2D interaction by providing more natural and intuitive techniques such as 2D translation, scaling and rotation, it has not been considered if and how these concepts can be extended to 3D multi-touch interfaces. The challenge of 3D interaction with stereoscopically displayed objects using a multi-touch user interface has only been considered rudimentarily. Multi-touch surfaces can be realized by using different technologies, ranging from capacitive sensing to video analysis of infrared or full colour video images [9-13]. Recently, the FTIR (frustrated total internal reflection) technology and its cheap footprint [10] (being a camera based multi-touch solution) has accelerated the usage of multi-touch. With today's technology it is now possible to apply the basic advantages of bi-manual interaction [14-19] to any suitable domain. Multi-touch surfaces can be easily integrated into systems supporting interaction of multiple users with two- dimensional data sets. Bill Buxton gives a comprehensive overview of the history of development of multi-touch surface1. Another benefit of multi-touch technology is that the user does not have to wear inconvenient devices in order to interact in an intuitive way. The DoF are restricted by the physical constraints of the touch screen. In combination with autostereoscopic displays, such a system can avoid any instrumentation of the user, while providing an advanced user experience. However, the benefits and limitations of using multi-touch in combination with stereoscopic display have not been examined in-depth and are not well understood [20].

Only some researchers have addressed the problem of 3D interaction on a $2 \mathrm{D}$ surface. Grossman et al. [21] presented a suite of gestural interaction techniques for use with a spherical 3D volumetric display. However, in this setup the user can already use 3D inputs on a touch sphere in comparison to available inputs on a twodimensional plane. To allow interactions in the 3D space Benko et al. [22] introduced the Balloon Selection, a 3D interaction technique that is modelled after the real-world metaphor of manipulating a helium balloon attached to a string. Balloon Selection allows for precise 3D selection in the volume above a tabletop surface by using multiple fingers on a multi-touch sensitive surface in conjunction with a $3 \mathrm{D}$ tracked glove. Although, this technique enables two-and-a-half-dimensional $(2.5 \mathrm{D}$ is an informal term used to describe visual phenomena which is actually $2 \mathrm{D}$ with $3 \mathrm{D}$ looking graphics) selection, real 3D interaction has not been considered in their work.

In this paper we discuss the challenges for iMUTS-based interaction paradigms, and present two new interaction techniques, which underline their benefits (see figure 1). The remainder of this paper is structured as follows: First we discuss the general interaction problem with interscopic data in Section 2. In Section 3, we describe two 
new multi-touch interactions metaphors that are best suited for multi-touch input and that can be interfaced by the described iMUTS setup. Section 4 describes the iMUTS technical setup in detail. Section 5 presents an initial evaluation of the presented concepts. Finally, Section 6 concludes the paper and gives an overview about future work.

\section{Interaction Challenges}

While it appears quite obvious to use multi-touch for 2D interaction, one might argue that it is not useful to limit interaction with $3 \mathrm{D}$ data to a $2 \mathrm{D}$ touch surface. As mentioned in the introduction, the usage of complex VR systems still requires much user instrumentation, and it is still quite complex to interact in the $3 \mathrm{D}$ space [1]. And as a matter of fact, 2D interaction devices usually supporting only two DoFs [6,7] are still in widespread use, although they are not the optimal devices for 3D interaction. These both facts motivated us to consider multi-touch interfaces with respect to the requirements of current 3D graphical applications.

\subsection{Taxonomy of User interfaces}

In Figure 2, a taxonomy is illustrated which classifies iMUTS paradigms within the broad field of graphical user interfaces. This classification includes conventional desktop systems as well as VR and current multi-touch environments. The taxonomy model is based on a coordinate system involving three axes representing generality, presence and nativeness. Generality captures the variety of interaction tasks that can be performed with the corresponding user interface, presence measures the degree to which a user believes the virtual environment (VE) is part of the physical surrounding or vice versa, and nativeness denotes how inartificial the user interface appears with respect to the required instrumentation. Current graphical user interfaces can be classified according to these characteristics. For example, as mentioned in Section 1,

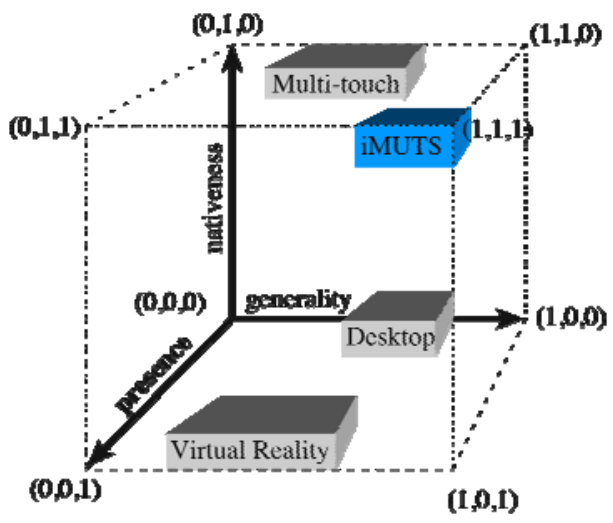

Fig. 2. Taxonomy of different user interface paradigms and the integration of GUIs based on iMUTS. The three axes representing generality, presence and nativeness. 
desktop-based environments are most appropriate for 2D interaction tasks, but are not optimal for immersive 3D interaction. Hence they are specialized rather than general in this case. Furthermore, virtual scenes are usually displayed monoscopically in desktop-based environments, with only a small field of view and no-head tracking is supported; therefore, the user's sense of presence is often lower in this case. Although interaction with traditional input devices is quite natural, the instrumentation with keyboard and/or mouse is less natural than using the hands directly as it is possible with multi-touch user interfaces (see figure 2). For these reasons, desktop systems are mapped to the area close to the origin $(0,0,0)$. VR systems increase presence and are suitable for 3D interaction, but lack support for 2D interaction (cf. Section 1). Furthermore, instrumentation by haptic devices such as data gloves and/or immersive display systems such as head-mounted-displays leads to non-native interaction. Hence we arrange VR systems similar to desktop-based environments, but closer to the front bottom edge. Current multi-touch user interfaces are suitable for ordinary desktop tasks, they provide the same sense of presence, but they also allow native interaction by the hands. Hence they are arranged above desktop systems showing increased nativeness. We believe that the iMUTS has the potential to increase presence (e.g., due to stereoscopic projection) in comparison to existing multi-touch user interfaces, and that they will support intuitive $2 \mathrm{D}$ as well as $3 \mathrm{D}$ interactions while not requiring additional instrumentation of the user. Hence, iMUTS user interfaces may meet most requirements for current GUIs. We admit that this taxonomy is neither perfect nor universal, but it points out some of the benefits that we believe iMUTS paradigms may provide.

To summarize: with iMUTS, humans may interact spontaneously while no heavy instrumentation is required. Further advantages of iMUTS are:

- Multiple Users: iMUTS easily supports the interaction between different users.

- Intuitivity: People have developed sophisticated skills for sensing and manipulating their physical environments [23]. iMUTS supports these skills.

- Spontaneity: User can switch between different tasks spontaneously.

- Costs: The presented iMUTS setup can be realized with low costs.

As mentioned in Section 1, interaction with stereoscopically displayed objects is still a challenging task [1], in particular when the interaction is restricted to a $2 \mathrm{D}$ touch surface. This is the main limitation of an iMUTS. In the following section, we explain this issue and discuss solutions, which have proven their usability in other domains.

\subsection{Parallax Problems}

In order to display graphical content stereographically, two half-images have to be generated, i.e., one for each eye. When using stereoscopic projection, a 3D impression occurs due to binocular disparity, which means that objects in space are projected to different positions on the screen. The corresponding horizontal displacement results in essentially three different stereoscopic display paradigms: negative, zero and positive parallax. Objects having zero parallax are displayed monoscopically and therefore are ideally suited for multi-touch interaction (see green-colored box in Figure 3). Both eyes perceive the same image, which causes a two-dimensional impression. As mentioned in the introduction, for such a situation multi-touch interfaces have 

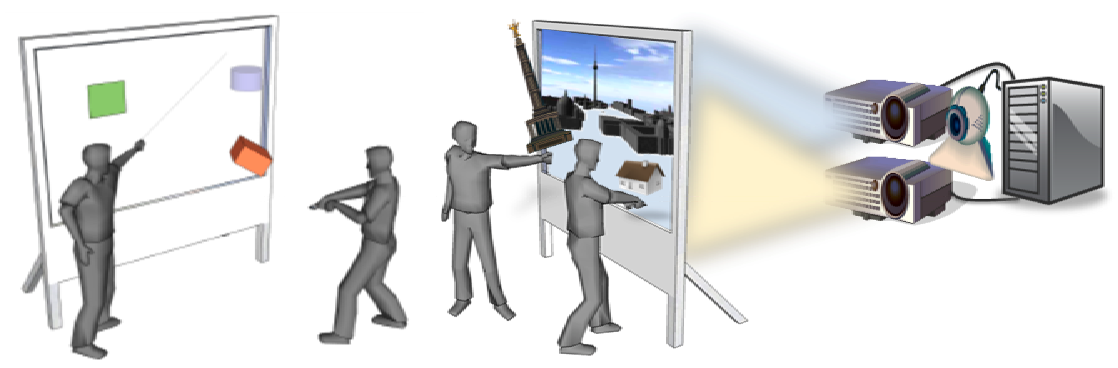

Fig. 3. (left) Illustration of two users interacting with stereo content, as well as monoscopic content (green rectangle: zero parallax, orange-colored box: negative parallax, purple-colored cylinder: positive parallax), and (right) during an interaction with a city planning application on an interscopic multi-touch surface (iMUTS))

considerable potential to enhance the interaction process, in particular when 2D manipulations are intended. Objects with positive parallax appear behind the touch screen and therefore cannot be accessed directly due to the screen limiting the reach of the user (see purple-coloured cylinder in Figure 3). This is a problem for any kind of direct interaction in stereoscopic environments, and several approaches address this issue $[1,24]$, e.g., distant objects behind the screen can be selected by casting a virtual ray [25]. Objects displayed with negative parallax appear in front of the projection screen (see orange-coloured box in Figure 3) When the user wants to interact with such objects by touching, s/he is limited to touching the area behind the objects since multi-touch screens capture only direct contacts. Therefore, the user virtually has to move fingers or her/himself through virtual objects, and the stereoscopic projection is disturbed. Consequently, immersion may get lost. This problem is a common issue known from two-dimensional representation of the mouse cursor within a stereoscopic image. While the mouse cursor can be displayed stereoscopically on top of stereoscopic objects [8], movements of real objects in the physical space, e.g., the user's hands, cannot be constrained such that they appear only on top of virtual objects. Therefore direct grabbing of objects in front of the touch screen is not possible, and moreover, the hands may interfere with the stereoscopic effect. When objects are displayed with negative parallax, any input devices tracked with six DoF support direct interaction. However, we admit that this requires an instrumentation of the user again. Hence, multi-touch devices in combination with image-based gesture recognition may be more appropriate, but have not been used in our setup until now.

\section{Interaction Metaphors}

As mentioned above, 3D visualization applications combine two-dimensional with three-dimensional content. While 3D data has the potential to benefit from stereoscopic display, visualization and interaction with $2 \mathrm{D}$ content should be restricted to two dimensions [2]. In this section, we discuss aspects that have to be taken into account when designing a multi-touch user interface for interscopic interaction. Therefore, we present interaction metaphors with interscopic data for a city-planning scenario as well as for medical volume deformation. The described metaphors are not 
limited to the city or medical domain, but can be applied also to other scenarios. Both interaction concepts are motivated by the work of Wilson [26], which combines multi-touch sensing with a physics engine. In the first scenario, users can manipulate a virtual window analog to a plate on a ball and socket joint. This enables an intuitive way of travelling through a 3D city model. Although such 3D city models are usually represented by polygonal descriptions, the iMUTS interaction paradigms are not limited to polygonal data. To underline that also other formats, such as raster-based and volumetric data representations can be interfaced with iMUTS-based interaction paradigms, we present a second interaction metaphor with which users can directly manipulate a volume dataset by multi-touch deformation.

\subsection{Windows on the World Interaction with Interscopic Data}

During our long-term cooperation with the urban development, city planning and transport planning office as well as the land surveying and land registry office of the city of Münster, Germany, the needs for intuitive interfaces for the urban planning process (see Figure 4 (left)) became obvious. City planning tasks are highly cooperative and dynamic problems that involve several users with different expertise according to Broll [27], e. g., architects, planners, designers, politicians, potential home buyers, etc. Due to their naturalness, multi-touch user interfaces have considerable potential to increase performance and creativity for such a setup. All co-operators can be involved from the beginning and can design simultaneously. While many research groups address visualization techniques, for example for seismic exploration data, less effort has been undertaken in order to provide intuitive 3D user interfaces for such data sets.

For iMUTS, we designed a new interaction metaphor to easily navigate through a stereoscopically displayed 3D city model. The Windows on the World navigation metaphor allows users to easily navigate through a virtual city model displayed in an application with 2D graphical user interface elements and a 3D virtual world. This metaphor should not be confused with the work of Feiner et al. [28], a prototype heads-up window system intended for use in a 3D environment. Our metaphor is
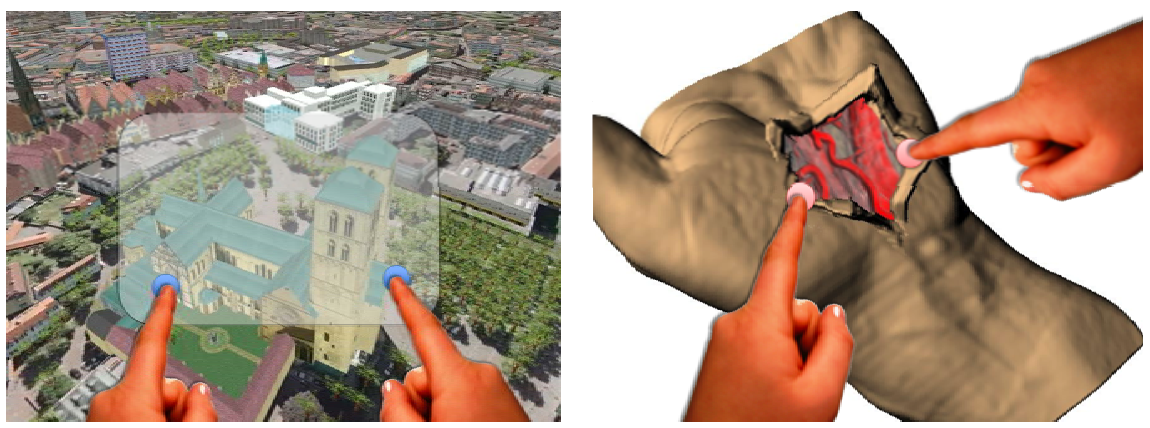

Fig. 4. Two example applications which can be interfaced by novel user interfaces based on the iMUTS paradigms: (left) navigating through a virtual city model with the Window of the World metaphor, and (right) multi-touch deformation of volume data in a medical scenario 
based on a virtual analogy of a physical plate that is attached to a ball and socket joint. Hence, in analogy to such a physical setup in our approach users can manipulate a virtual window overlaid on the virtual city or landscape model. The window is displayed with zero parallax so the user has the feeling that she can manipulate this virtual window. By touching the window the user can control the point of view, e.g., pressing on the bottom of the virtual window will cause that the user will look up (see Figure 4 (left)). By touching the top window edge, the user will look down. Turning the window to the left will cause a right rotation and vice versa. By pushing the middle of the window with both hands, the user navigates forward through the virtual model. The user can also rotate the virtual window to rotate the view. Zooming the window will enlarge the virtual display; thereby widening the opening angle of the virtual camera. The metaphor is independent from the device via which the user gives touch input. In addition to FTIR-based multi-touch surfaces, the user can use mobile devices that are capable to track multiple touches simultaneously. We have tested the metaphor with the described FTIR-based setup as well as with Apple's iPhone. The landscape planning application is based on NASA's World Wind2 using the Javabased SDK. The NASA World Wind visualization platform is open source and comes with a rich SDK for data set and interface customization.

\subsection{Volume Deformation with Interscopic Data}

Volume deformation is one example technique that allows users to modify volume data by means of warping. We have developed techniques for deforming volumetric data sets based on physically inspired approaches. In our system, user interaction mainly consists of two operations: specifying and applying a cut, as well as deforming the newly cut object. These operations are usually applied sequentially and can therefore be handled independently during the interaction process. The common problem is that a three-dimensional object is transformed, while the user only sees a twodimensional projection onto the screen and is using two-dimensional input devices. We implemented this deformation originally for mouse-based interaction. Due to the reduced degrees of freedom, the user interface is simplified by providing a small set of predefined cuts specified by cutting templates, which may be resized and positioned freely within the scene. These templates share some resemblance with a cookie cutter, with the main difference that they do not start cutting as soon as they touch an object, but only when the user explicitly initiates the cut. This is a typical example, in which mouse-based as well as 3D interaction is limited and does not support the mental model of a deformation.

For this particular kind of interaction, multi-touch has considerable potential to provide a natural as well as effective user interface. Figures 5 (right) and 4 (right) show an illustration of this approach. The user can intuitively define the deformation by means of warping the volume data set at certain points simply by touching and moving with multiple inputs simultaneously, for example by using the edge or part of the palm of one or both hands. We have implemented this volume deformation with stereoscopic projection, which we restricted to almost zero parallax, i.e., parts of the volume were slightly above and below the touch surface. This kind of projection provides a sufficient three-dimensional visualization of the volume data set, while the 

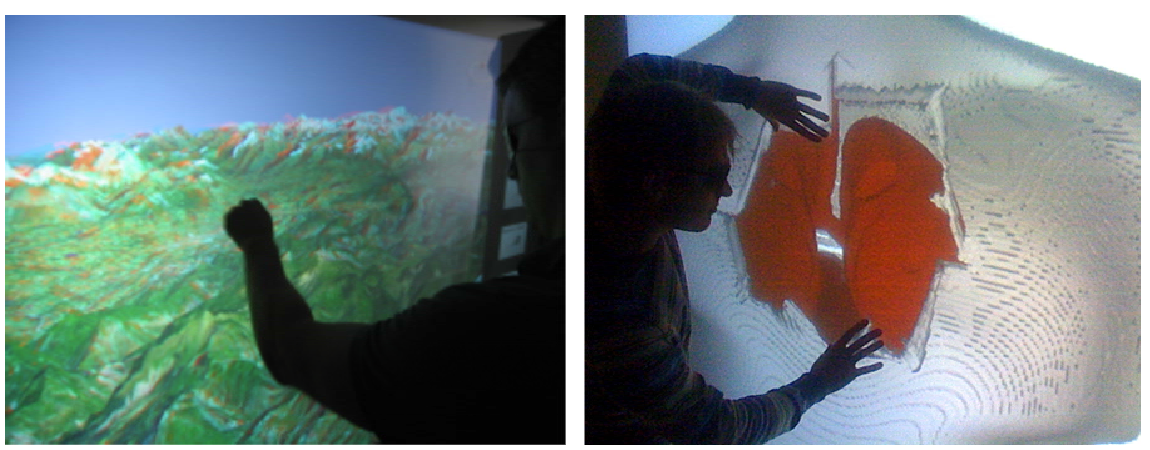

Fig. 5. Two examples of applications interfaced by the iMUTS interaction paradigms. (Left) a user interacts in a landscape-planning scenario on an FTIR multi-touch wall with an anaglyphbased stereoscopic projection. (Right) a user performs volume deformation in a medical scenario using a large FTIR-based passive back projection iMUTS.

interaction via the two-dimensional surface with the 3D data can still be performed without requiring additional tracked input devices.

Our physical-inspired implementation of the deformation of volume data sets exploits the 3D ChainMail algorithm in combination with a GPU-based ray-casting renderer in order to ensure interactive frame rates. We have experienced that this kind of physics-based interaction supports users because their knowledge from the realworld can be transferred easily to virtual interaction tasks $[8,26]$.

\section{4 iMUTS - Technical Setup}

In this section, we describe the system components of our iMUTS setup and discuss challenges for user interfaces based on such an interscopic multi-touch environment. There are several ways of combining multi-touch screens with a stereoscopic display in order to improve depth perception while providing intuitive interaction paradigms without further instrumentation of the user. Our multi-touch interscopic wall prototype is based on the FTIR principle introduced by Han [10]. We use a $180 \mathrm{~cm} \times 220 \mathrm{~cm}$ and $1.1 \mathrm{~cm}$ thick sheet of acrylic, whose edges have been polished clear, as an optical waveguide. This sheet is edge-lit by high-power infrared LEDs, which are placed directly against the polished edges so as to maximize coupling into total internal reflection, while a digital video camera equipped with a matching band-pass filter is mounted orthogonally. Total internal reflection keeps the light trapped within the sheet, except at points where it is frustrated by some object (e. g. finger) in optical contact, causing light to scatter out through the sheet towards the camera, a PointGrey Dragonfly23. The acrylic plate was mounted onto a wall and a wide-angle lens attached to the PointGrey Dragonfly 2 equipped with a matching infrared band-pass filter was mounted orthogonally at a two-meter distance. For display, an HKS rear projection screen is used and attached behind acrylic plate. Only simple image processing operations (rectification, background subtraction, noise removal, and connected components analysis) are required for each frame, and for that a Java multitouch library4, developed at the Deutsche Telekom Laboratories, has been used and 
extended. The library is released under the GNU Public License. It contains a set of common algorithms designed to work with any multi-touch system, such as routines to label connected components and track features. By using an application layer, it is easy to manipulate objects and transform (position, rotate, scale) them. The library also comes with a module for accessing cameras (currently just the Point-Grey Dragonfly2 is supported), and the library is able to stream the data into the TUIO protocol [29], the de facto standard for broadcasting multi-touch events over the network. Video is captured as 8-bit monochrome at $30 \mathrm{fps}$ at a resolution of $1024 \times 768$ (corresponding to $2,1 \mathrm{~mm} 2$ precision on the surface). For stereoscopic projection we have tested two different setups landscape planning and volume deformation in a medical scenario:

- a passive stereoscopic back projection with an FTIR based interactive wall (see Figure 5 (right)),

- $\quad$ and a simple anaglyph-based stereoscopic projection again based on an FTIR multi-touch wall (see Figure 5 (left)).

The passive stereoscopic projection screen is illustrated in Figure 3. Two DLP projectors with a resolution of $1248 \times 1024$ provide half images for the left and right eye of the user. The half images are linearly filtered such that users have to wear corresponding polarized glasses. In the case of the anaglyph mode, the half images can be displayed by one projector with a resolution of $1920 \times 1080$. Colour masks applied to the rendering processes mask both half images such that the user can separate the half images with anaglyph glasses. For both setups the images are rendered by a computer with Intel dual-core processors, 4 GB of main memory and an nVidia GeForce 8800 GTX for rendering purposes.

\section{Initial Evaluation}

We conducted an initial user study to compare the subjects' self-reported experiences when interacting with interscopic multi-touch surfaces in comparison to traditional multi-touch surface. 2 female and 6 male subjects participated in the study (ages 21 33, mean 23.9 years). All subjects were members of the Department of Geoscience at university of Münster, Germany and familiar with landscape planning tasks. The study was set up as a within-subject design, where all participants had to perform the following task in two different conditions (interscopic multi-touch display and multitouch surface): subjects had to judge the landslide risk in a certain region in the Alps. The data was displayed in anaglyph stereoscopic mode in the interscopic condition and the Windows on the World Interaction metaphor was used. In the "pure" multitouch condition the Windows on the World Interaction metaphor was also used but the data was displayed directly on the interactive surface. The subjects had to explore a certain region by navigating to different viewports, to make a judgment about the region with the highest landslide risk. After the actual test, subjects were asked to rate both techniques by filling out a modified version of the "user interface evaluation questionnaire" of ISO 9241-9 with only a single Fatigue (seven-point rating; higher scores denote a better rating). The total time of the experiment was about 15 minutes for each participant. 


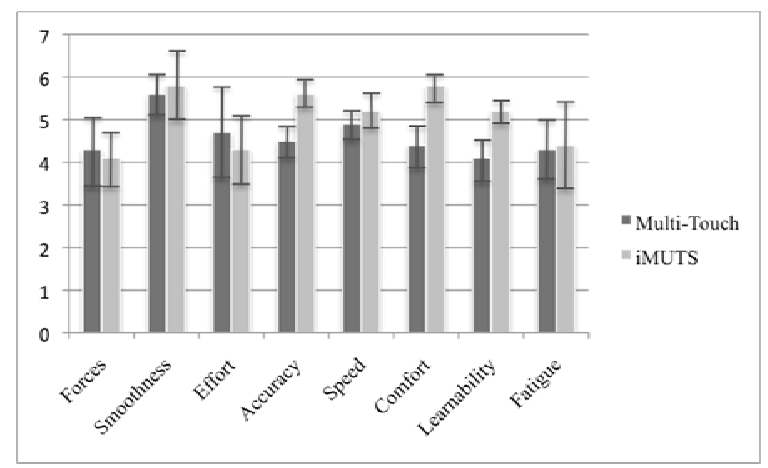

Fig. 6. Results of the user interface evaluation questionnaire ISO 9241-9 (seven-point rating; higher scores denote a better rating)

The answers varied strongly between subjects, which is reflected in the large confidence interval. Figure 6 shows that subjects rated accuracy with 5.6 on average when using the iMUTS setup. This is an increase of $20 \%$ in comparison to the evaluation of the traditional multi-touch map interaction. Only the differences in the categories accuracy, comfort and learnability are significant at the 5\% level. In general, from the user comments they preferred the interscopic, because they felt more embedded in the scenario and it was easier to get depth information, which is important for evaluating landslide risk. They tended to perform the task faster, because they could perform actions (e.g. panning and zooming) more accurately and navigate more easily to the spots they wanted to inspect than with a pure multi-touch system. In general, the users had no problem performing the Windows on the World Interaction, after basically getting the idea behind the interaction metaphor. Overall, the users preferred the iMUTS system and they gave us comments like: "It feels like I am there." or "Hopefully the fieldwork will be as easy as manipulating the whole world from my window board". Both statements underline the argument that the user believes the iMUTS is part of the physical surrounding or vice versa and supporting presence.

\section{Conclusion}

In this paper, we have introduced a new interscopic multi-touch paradigm that combines traditional 2D interaction performed in monoscopic mode with 3D interaction and stereoscopic projection. We discussed challenges and potentials for the use of multi-touch interfaces for the interaction with interscopic data. In addition, we have introduced two different systems of iMUTS:

- a passive back projection with an FTIR-based interactive wall (see Figure 5),

- $\quad$ and a simple anaglyph-based stereoscopic projection again based on an FTIR multi-touch wall.

For both applications, we have highlighted two new multi-touch interaction metaphors, which benefit from multi-touch in combination with monoscopic or stereoscopic 
projection: the Window on the World navigation method and direct volume deformation. Furthermore, we have presented two different application scenarios, i.e., city \& landscape planning and medical exploration that can be interfaced with user interfaces based on iMUTS. We believe that iMUTS has great potential to fill the gap between WIMP and VR systems to form the basis of the next generation of $2 \mathrm{D}$ and $3 \mathrm{D}$ user interfaces. They provide intuitive, fast and spontaneous access to 3D information for multiple users at low costs without requiring user instrumentation. Moreover, we have outlined some challenges and limitations that might occur in such scenarios. Currently, multi-touch walls are horizontally or vertically mounted. VR-based display devices such as the responsive workbench allow users to turn the display from horizontal to vertical. In contrast to vertical multi-touch surfaces, horizontal ones provide the possibility to place physical objects on the surface 5. For some application domains, it might be beneficial to present the stereoscopic content on a non-planar surface like Microsoft's sphere [30]. With respect to the surface geometry, it might be possible that in some areas objects may not be placed due to instabilities caused by gravitation. The special problem of interaction with stereoscopic data displayed with negative parallax has not been addressed in detail within the scope of this paper and will be considered in future iMUTS. However, in such a case, the user can use arbitrary input devices that can be tracked with six degrees of freedom. Another solution might be to allow a user to interactively change the parallax of objects by using a mobile device attached to the user's body as a "soft slider". If the touch surface is portable, the screen can be moved through the VE (analog to the 3D Window on the World metaphor) until desired objects are displayed with zero or negative parallax and interaction can be performed as described above. In order to provide stereoscopic images without the need for glasses, in future work we will set up a transparent multi-touch wall in front of an autostereoscopic display. Hence, natural and intuitive interfaces can be provided which do not require any instrumentation, but support 3D interaction. In addition we will carry out further user studies with this next prototype.

\section{References}

1. Bowman, D., Kruijff, E., LaViola, J., Poupyrev, I.: 3D User Interfaces: Theory and Practice. Addison-Wesley, Reading (2004)

2. Balakrishnan, R.: A Grant of 3D. Why aren't we using 3D user interfaces, and will we ever? (3D User Interfaces Keynote) (2006)

3. Myers, B.A.: A taxonomy of window manager user interfaces. IEEE Comput. Graph. Appl. 8(5), 65-84 (1988)

4. Chun, W.S., Napoli, J., Cossairt, O.S., Dorval, R.K., Hall, D.M., Purtell II, T.J., Schooler, J.F., Banker, Y., Favalora, G.E.: Spatial 3-D Infrastructure: Display-Independent Software Framework, High-Speed Rendering Electronics, and Several New Displays. In: Stereoscopic Displays and Virtual Reality Systems XII, vol. 5664, pp. 302-312 (2005)

5. Conner, D.B., Snibbe, S.C., Herndon, K.P., Robbins, D.C., Zeleznik, R.C., van Dam, A.: Three-Dimensional Widgets. In: Symposium on Interactive 3D Graphics (1992)

6. Hanson, A.J., Wernert, E.: Constrained 3D Navigation with 2D Controllers. In: Proceedings of Visualization 1997, pp. 175-182. IEEE Computer Society Press, Los Alamitos (1997) 
7. Salzman, T., Stachniak, S., Stürzlinger, W.: Unconstrained vs. Constrained 3D scene manipulation. In: Nigay, L., Little, M.R. (eds.) EHCI 2001. LNCS, vol. 2254, p. 207. Springer, Heidelberg (2001)

8. Steinicke, F., Ropinski, T., Bruder, G., Hinrichs, K.: Interscopic User Interface Concepts for Fish Tank Virtual Reality Systems. In: Proceedings of the Virtual Reality, pp. 27-34 (2007)

9. Dietz, P., Leigh, D.: DiamondTouch: a multi-user touch technology. In: Proceedings of the 14th annual ACM symposium on User interface software and technology, pp. 219-226 (2001)

10. Han, J.Y.: Low-cost multi-touch sensing through frustrated total internal reflection. In: UIST 2005: Proceedings of the 18th annual ACM symposium on User interface software and technology, pp. 115-118. ACM, New York (2005)

11. Malik, S., Laszlo, J.: Visual touchpad: a two-handed gestural input device. In: Proceedings of the 6th international conference on Multimodal interfaces, pp. 289-296 (2004)

12. Peltonen, P., Kurvinen, E., Salovaara, A., Jacucci, G., Ilmonen, T., Evans, J., Oulasvirta, A., Saarikko, P.: It's mine, don't touch!: interactions at a large multi-touch display in a city centre. In: CHI 2008: Proceeding of the twenty-sixth annual SIGCHI conference on Human factors in computing systems, pp. 1285-1294. ACM, New York (2008)

13. Schöning, J., Brandl, P., Daiber, F., Echtler, F., Hilliges, O., Hook, J., Löchtefeld, M., Motamedi, N., Muller, L., Olivier, P., Roth, T., von Zadow, U.: Multi-touch surfaces: A technical guide. Technical report, Technical University of Munich (2008)

14. Buxton, W., Myers, B.: A study in two-handed input. In: Proceedings of the SIGCHI conference on Human factors in computing systems, pp. 321-326 (1986)

15. Lee, S., Buxton, W., Smith, K.: A multi-touch three dimensional touch-sensitive tablet. ACM SIGCHI Bulletin 16(4), 21-25 (1985)

16. Matsushita, N., Rekimoto, J.: HoloWall: designing a finger, hand, body, and object sensitive wall. In: Proceedings of the 10th annual ACM symposium on User interface software and technology, pp. 209-210 (1997)

17. Epps, J., Lichman, S., Wu, M.: A study of hand shape use in tabletop gesture interaction. In: CHI 2006: CHI 2006 extended abstracts on Human factors in computing systems, pp. 748-753. ACM, New York (2006)

18. Wu, M., Balakrishnan, R.: Multi-finger and whole hand gestural interaction techniques for multi-user tabletop displays. In: Proceedings of the 16th annual ACM Symposium on User Interface Software and Technology, pp. 193-202 (2003)

19. Schöning, J., Hecht, B., Raubal, M., Krüger, A., Marsh, M., Rohs, M.: Improving Interaction with Virtual Globes through Spatial Thinking: Helping users Ask "Why?". In: IUI 2008: Proceedings of the 13th annual ACM conference on Intelligent User Interfaces. ACM Press, New York (2008)

20. Steinicke, F., Schöning, J., Krüger, A., Hinrichs, K.: Multi-touching cross-dimensional data: towards direct interaction in stereoscopic display environments coupled with mobile devices. In: AVI 2008: Workshop on designing multi-touch interaction techniques for coupled private and public displays (2008)

21. Grossman, T., Wigdor, D., Balakrishnan, R.: Multi-finger gestural interaction with 3d volumetric displays. In: SIGGRAPH 2005: ACM SIGGRAPH 2005 Papers, pp. 931-931. ACM, New York (2005)

22. Benko, H., Feiner, S.: Balloon selection: A multi-finger technique for accurate low-fatigue 3d selection. 3D User Interfaces (2007)

23. Ullmer, B., Ishii, H.: Emerging frameworks for tangible user interfaces. IBM Systems Journal 39(3), 915-931 (2000) 
24. Pierce, J., Forsberg, A., Conway, M., Hong, S., Zeleznik, R., Mine, M.: Image Plane Interaction Techniques in 3D Immersive Environments. In: ACM Symposium on Interactive 3D Graphics, pp. 39-44 (1997)

25. Mine, M.: Virtual Environments Interaction Technqiues. Technical Report TR95-018, UNC Chapel Hill Computer Science (1995)

26. Wilson, A.D., Izadi, S., Hilliges, O., Garcia-Mendoza, A., Kirk, D.: Bringing physics to the surface. In: UIST 2008: Proceedings of the 21st annual ACM symposium on User interface software and technology, pp. 67-76. ACM, New York (2008)

27. Broll, W., Lindt, I., Ohlenburg, J., Wittkämper, M., Yuan, C., Novotny, T., Fatah, A., Mottram, C., Strothmann, A.: Arthur: A collaborative augmented environment for architectural design and urban planning. Journal of Virtual Reality and Broadcasting (JVRB) 1(1), 1-10 (2004)

28. Feiner, S., MacIntyre, B., Haupt, M., Solomon, E.: Windows on the world: 2D windows for 3D augmented reality. In: Proceedings of the 6th annual ACM symposium on User interface software and technology, pp. 145-155. ACM, New York (1993)

29. Kaltenbrunner, M., Bovermann, T., Bencina, R., Costanza, E.: TUIO: A protocol for tabletop tangible user interfaces. In: Proc. of the The 6th Intl. Workshop on Gesture in HumanComputer Interaction and Simulation (2005)

30. Benko, H., Wilson, A.D., Balakrishnan, R.: Sphere: multi-touch interactions on a spherical display. In: UIST 2008: Proceedings of the 21st annual ACM symposium on User interface software and technology, pp. 77-86. ACM, New York (2008) 